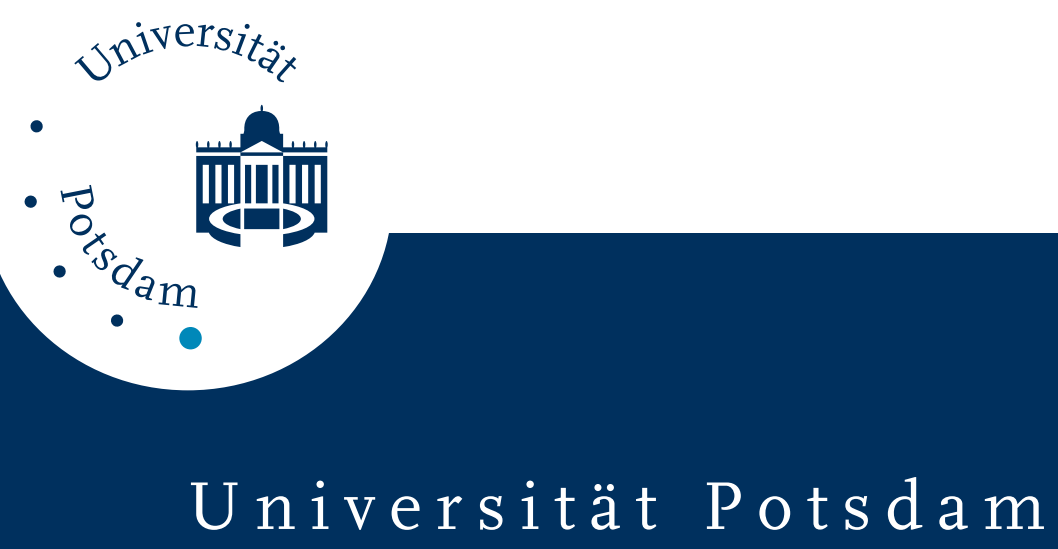

Stanislaw A. Rozanski, Friedrich Kremer, Peter Köberle, André Laschewsky

\title{
Relaxation and charge transport in mixtures of zwitterionic polymers and inorganic salts
}

first published in:

Macromolecular Chemistry and Physics, 196 (1995) S. 877 - 890,

ISSN 1521-3935

DOI 10.1002/macp.1995.021960316

Postprint published at the Institutional Repository of Potsdam University:

In: Postprints der Universität Potsdam

Mathematisch-Naturwissenschaftliche Reihe ; 93

http://opus.kobv.de/ubp/volltexte/2009/1743/

http://nbn-resolving.de/urn:nbn:de:kobv:517-opus-17438

Postprints der Universität Potsdam

Mathematisch-Naturwissenschaftliche Reihe ; 93 


\title{
Relaxation and charge transport in mixtures of zwitterionic polymers and inorganic salts
}

\author{
Stanislaw A. Rozanski ${ }^{\text {a) }}$, Friedrich Kremer* \\ Institut für Physik, Universität Leipzig, Linnéstr. 5, D-04103 Leipzig (Germany)
}

Peter Köberle

Institut für Org. Chemie, Universität Mainz, Becher-Weg 18-20, D-55099 Mainz (Germany)

André Laschewsky

Université Catholique de Louvain, Département de Chimie, Place L. Pasteur 1, B-1348 Louvain-la-Neuve (Belgium)

(Received: July 21, 1994)

\section{SUMMARY:}

Dielectric spectroscopy is employed to analyze the molecular dynamics and the charge transport in mixtures of zwitterionic polymers of the type poly $\{3-[N-(\omega$-methacryloyloxyalkyl $)$ $N, N$-dimethylammonio]propanesulfonate $\}$ with sodium iodide in the frequency range of $10^{2} \mathrm{~Hz}-10^{7} \mathrm{~Hz}$ and in the temperature range of $110 \mathrm{~K}-400 \mathrm{~K}$. The amount of inorganic salt added varies from $0-200$ mol- $\%$ relative to the number of zwitterionic groups present in the polymer, contributing strongly to the conductivity. One relaxation process is observed whose relaxation rate depends strongly on the length of the aliphatic spacer between the polymethacrylate main chain and the zwitterionic group. Exhibiting an Arrhenius-like temperature dependence with activation energy $E_{\mathrm{A}}=47 \mathrm{~kJ} / \mathrm{mol}$, this relaxation process is assigned to fluctuation of the quaternary ammonium groups in the side chains. At higher temperatures, the dielectric properties and the conductivity are primarily dominated by the mobile inorganic ions: conductivity strongly depends on the salt concentration, showing a pronounced electrode polarization effect. The frequency and sait concentration dependences of the conductivity can be quantitatively described as hopping of charge carriers being subject to spatially randomly varying energy barriers. For the low-frequency regime and for the critical frequency marking the onset of the conductivity's dispersion, the Barton-Nakajima-Namikawa (BNN) relationship is fulfilled.

\section{Introduction}

Dielectric spectroscopy is one of the well established techniques in polymer science for studying molecular dynamics. The sensors in the determination of the molecular dynamics are the molecular dipoles. In polymer blends and block copolymers, relaxation processes become broadened in comparison to homogeneous systems. This can be used as a dielectrically determined miscibility criterium ${ }^{1)}$. In electrically conducting polymers, the frequency dependence and the temperature dependence of the complex dielectric function enable conclusions on the mechanisms of charge transport ${ }^{2}$.

\footnotetext{
a) Permanent address: Pl-64-920 Pila, ul. Sniadeckich 13B/7, Poland.
} 
One of the most characteristic properties of electrical conduction in disordered solids is the strong dispersion of the conductivity. The electrical characteristics of ionically conducting materials are often studied by ac techniques to avoid the necessity of developing non-blocking ion-conducting electrodes which would be needed for $\mathrm{dc}$ measurements. At low frequencies a constant conductivity is observed, while at higher frequencies the conductivity becomes frequency dependent, varying approximately as a power of the frequency. The increase in conductivity usually continues up to phonon frequencies. This behaviour is found in ionenes ${ }^{2,3)}$ and cationic polymer glasses ${ }^{4)}$, solid solution of betaine phosphate and betaine phosphite molecular crystals ${ }^{5)}$ or amorphous semiconductors ${ }^{6}$.

To describe the frequency and temperature dependences of the complex conductivity, a variety of mathematical approaches have been proposed emphasizing the power-law behaviour of $\sigma^{\prime}(\omega)$ which is regarded as fundamental ${ }^{7-9)}$. The most studied models for ac conduction in disordered solids are the hopping models ${ }^{10-15}$. However, a physical interpretation of these approaches is non-existent. It is still not clear which model for ac conduction is correct ${ }^{16-18)}$ and to what extent Coulomb interactions are important.

A particularly interesting case is given for zwitterionic polymers ${ }^{19-21)}$. In such polymers, all ions are fixed to the polymer, and thus low-molecular-weight counterions are missing. As each macromolecule bears the same number of cationic and anionic groups, the individual macromolecules are uncharged in spite of the high concentration of ionic groups present. The high density in dipolar units results in a number of specific properties of polyzwitterions ${ }^{22)}$ : very high glass transition temperatures, strong polarity and hygroscopy. Furthermore, these polymers are strongly interacting with low-molecular-weight cations and anions, both in solution and in bulk. In selected cases, these interactions enable the preparation of homogeneous mixtures of polyzwitterions with inorganic salts containing up to stoechiometric amounts of the salt. This behaviour was recently demonstrated for a number of zwitterionic poly(sulfobetaines) blended with $\mathrm{LiClO}_{4}, \mathrm{NaBr}$ or $\mathrm{NaI}{ }^{19,20,23,24-27)}$. Phase segregation is only observed in mixtures containing more than 125 mol- $\%$ of salt.

The dynamic and, in particular, the dielectric properties of zwitterionic polymers and their conductivity have been scarcely explored up to now ${ }^{23,28,26-30}$. Here, the frequency and temperature dependence of the dielectric functions of some poly\{3-[ $N$-( $\omega$-methacryloyloxyalkyl)- $N, N$-dimethylammonio]propanesulfonatejs and of some of their blends with $\mathrm{NaI}$ are measured by broadband dielectric spectroscopy. These studies allow one to investigate the underlying mechanisms of charge transport and the influence of the concentration of the inorganic salt on this process.

\section{Experimental part}

The synthesis of monomers and polymers, and the preparation of the blends with inorganic salts, have been described previously ${ }^{24)}$. All measurements were made below the calorimetric glass transition temperature, which is higher than the decomposition temperature of the polymers of ca, $473 \mathrm{~K}$.

For the dielectric measurements, polymers films were placed between two gold plated stainless steel electrodes (diameter: $20 \mathrm{~mm}$ ) that were pressed together by a micrometer screw. The polymer 
films with different $\mathrm{NaI}$ contents and with a thickness ranging between $40 \mu \mathrm{m}$ and $80 \mu \mathrm{m}$ were cast directly from $2 \mathrm{~mL} 1: 1(\mathrm{v} / \mathrm{v})$ mixtures of 2,2,2-trifluoroethanol with water. To provide good contact with the upper electrode of the sample condenser, a graphite foil (16 mm diameter) was placed on top of the polymer film. For the dielectric measurements $\left(10^{2}-10^{7} \mathrm{~Hz}\right)$, an impedance analyser (HP 4192A) was employed. The measurement system used a custom-made cryostat, which allowed to adjust the sample temperature between $100 \mathrm{~K}$ and $400 \mathrm{~K}$ by using a temperaturecontrolled nitrogen gas jet (stability $\pm 0,02 \mathrm{~K}$ ). The sample temperature was measured with a platinum temperature sensor mounted in one of the condenser plates (temperature resolution $\pm 0,01 \mathrm{~K}$ ). Prior to the measurements, the samples were dried in a nitrogen gas jet at $400 \mathrm{~K}$ until the resistivity reached a stable level.

\section{Results and discussion}

To elucidate the influence of inorganic salt concentration on the dielectric properties and conductivity in ionic side-chain polymers, we have studied several poly $[3-[N-(\omega-$ methacryloyloxyalkyl)- $N, N$-dimethylammonio]propanesulfonatejs at several concentrations of sodium iodide (Scheme 1).

Scheme 1. Chemical structure of the zwitterionic polymethacrylate poly\{ $3-[N-(\omega$-methacryloyloxyalkyl)- $N, N$-dimethylammonio]propanesulfonate) where $m=2(\mathbf{P 1}), m=6(\mathbf{P} 2)$, and $m=11$ (P3)

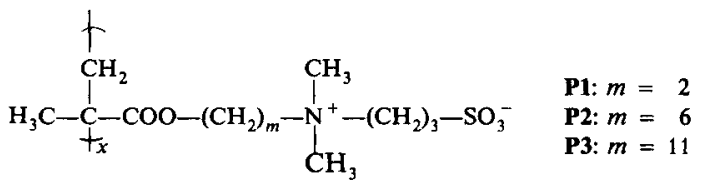

In polymers $\mathbf{P 2}$ and $\mathbf{P 3}$, the ionic groups are separated from the polymer backbone by long alkyl spacers ( $m=6$ and 11$)$. In contrast, in polymer P1 the ionic groups are attached closely to the backbone $(m=2)$. Hence, the comparison of the polymethacrylates with different spacers allows one to investigate the influence of the location of the betaine groups on the relaxation process and conductivity.

The dielectric properties of the salt-polyzwitterion mixtures are characterized by the superposition of a strong conductivity contribution and a dielectric relaxation process. Thus the analysis of the spectra has to be based on both the complex conductivity $\sigma^{*}(\omega)$ $=\sigma^{\prime}(\omega)+\mathrm{i} \sigma^{\prime \prime}(\omega)$ and the complex dielectric function $\varepsilon^{*}(\omega)=\varepsilon^{\prime}(\omega)+\mathrm{i} \varepsilon^{\prime \prime}(\omega)$, which are related to each other by:

$$
\sigma^{*}(\omega)=\text { i } \omega \varepsilon^{*}(\omega)
$$

For elevated temperatures $(>243 \mathrm{~K}$ ), the frequency dependence and the temperature dependence of the complex dielectric function (Fig. $1 \mathrm{a}, \mathrm{b}$ ) are dominated by a strong conductivity contribution. This gives rise to a steep increase in $\varepsilon^{\prime \prime}$ with decreasing 

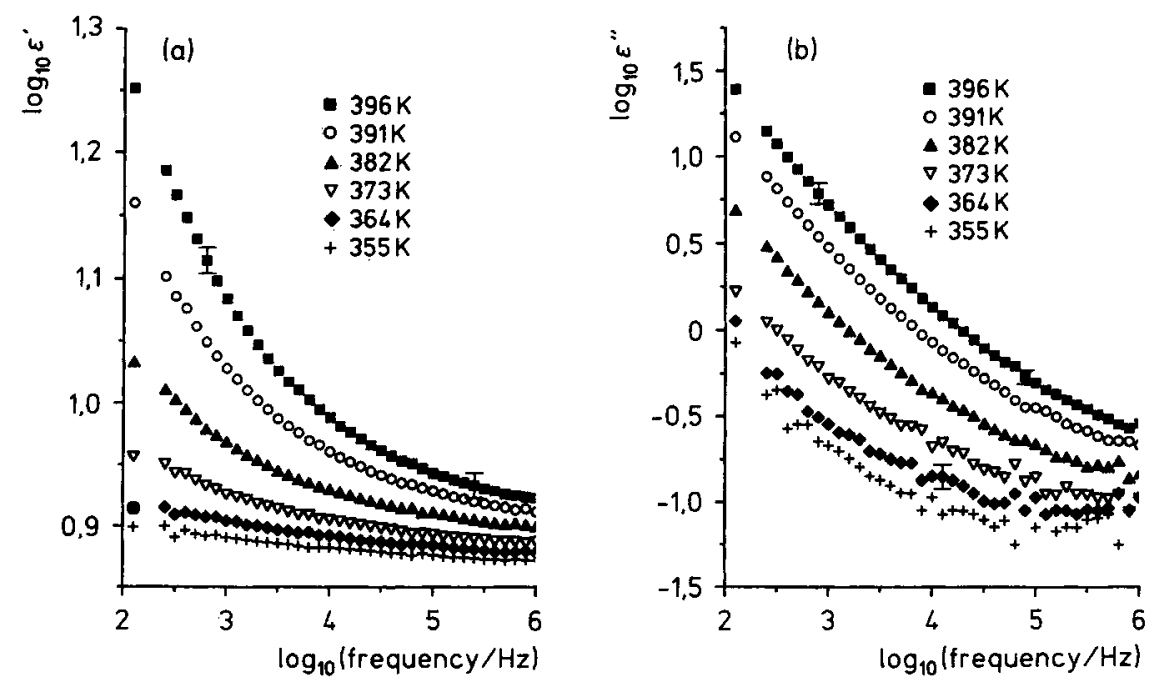

Fig. 1. (a) Real $\varepsilon^{\prime}$ and (b) imaginary $\varepsilon^{\prime \prime}$ parts of the complex dielectric function $\varepsilon^{*}(\omega)$ versus frequency for $\mathbf{P 3}$ at different temperatures. Typical error bars are indicated

frequency and increasing temperature (Fig. 1 b). The fact that $\varepsilon^{\prime}$ shows a similar frequency and temperature dependence as well proves that the charge carriers involved in charge transport are primarly of ionic origin, and hence cause a pronounced electrode polarization. This was also observed in glassy ionenes ${ }^{2)}$ and epoxide-amine addition polymers ${ }^{4)}$.

At lower temperatures $(<243 \mathrm{~K})$ one relaxation process occurs which was covered at high temperatures by the conductivity contribution. The maximum of $\varepsilon^{\prime \prime}$ shifts to higher frequencies with increasing temperature. To separate these two processes, the experimental data can be quantitatively analysed by a superposition of the conductivity contribution and the relaxation process. For the latter the generalized relaxation function according to Havriliak-Negami ${ }^{31}$ ) is used

$$
\varepsilon^{\prime \prime}(\omega)=\left(\sigma_{0} / \varepsilon_{0}\right) \omega^{(a-1)}+\operatorname{Im}\left\{\left(\varepsilon_{\mathrm{s}}-\varepsilon_{\infty}\right) /\left(1+(\mathrm{i} \omega \tau)^{\alpha}\right)^{\gamma}\right\}
$$

where $\sigma_{0}$ is a strongly temperature-dependent factor describing the dc conductivity, $a$ is a constant with values between 0,5 and 1 being, characteristic for hopping conduction ${ }^{10)}, \varepsilon_{\mathrm{s}}$ and $\varepsilon_{\infty}$ describe the values of $\varepsilon^{\prime}$ at the low frequency and high frequency side of the relaxation, respectively, and $\alpha$ and $\gamma$ are constants that determine the width and asymmetry of the relaxation time distribution. Fig. 2 shows the conductivity and the relaxation contributions to $\varepsilon^{\prime \prime}$ separated according to Eq. (2). Fit parameters for the relaxation contribution to the dielectric loss $\varepsilon^{\prime \prime}$ for salt-zwitterionic mixtures are shown in Tab. 1. 


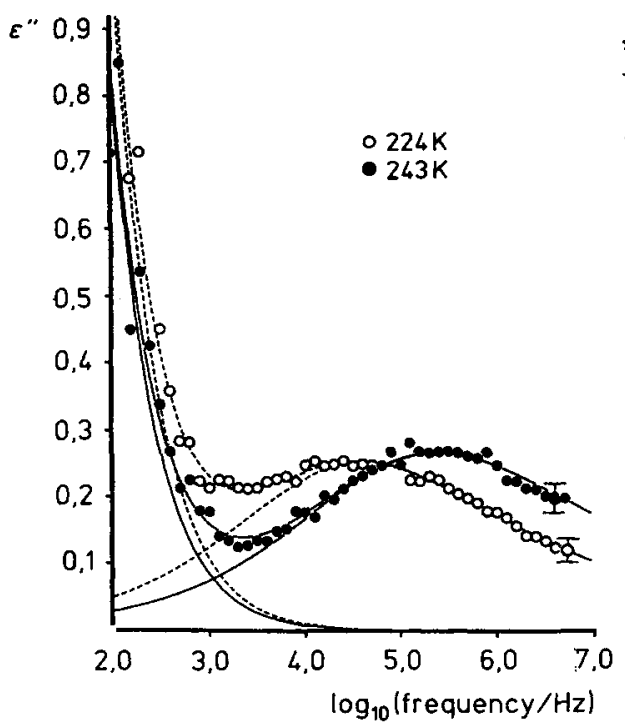

Fig. 2.

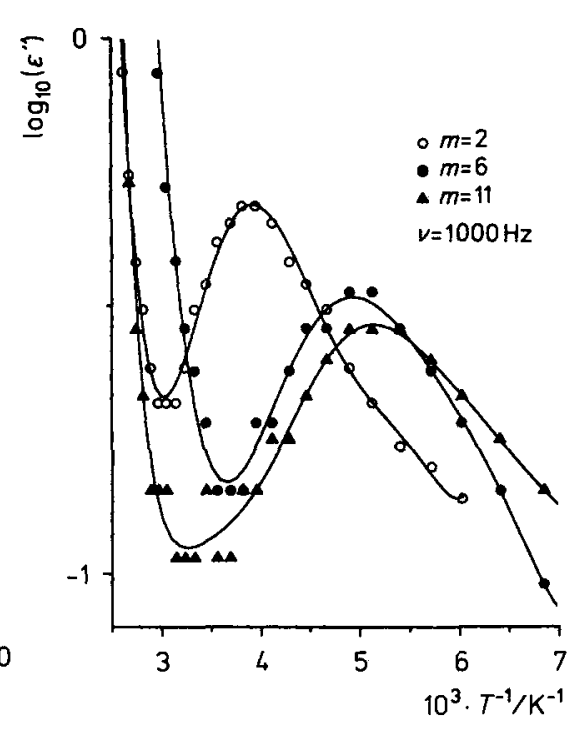

Fig. 3.

Fig. 2. Separation of the conductivity contribution and relaxation process according to Eq. (2) for P3. Temperature $T=224 \mathrm{~K}(---)$ and $T=243 \mathrm{~K}(\longrightarrow)$

Fig. 3. Temperature dependence of the dielectric loss $\varepsilon^{\prime \prime}$ for polymers P1-P3 for a frequency of $10^{3} \mathrm{~Hz} . m$ denotes the number of methylene groups separating the ester and the ammonium moiety (see Scheme 1)

The parameters $\alpha$ and $\gamma$, describing the relaxation time distribution, are nearly independent of the salt concentration. The relaxation process depends sensitively on the length of the spacer between the betaine group and the polymer backbone. The process is shifted to higher temperature (Fig. 3) with $\mathbf{P 3}<\mathbf{P 2}<\mathbf{P 1}$, i.e., with decreasing length of the alkyl spacer. In contrast, the amount of NaI added has only a negligible influence on this relaxation process (Fig. 4). The observed Arrhenius-type temperature dependence has an activation energy of $E_{\mathrm{A}}=47 \mathrm{~kJ} / \mathrm{mol}$. In that value and in its absolute frequency and temperature dependence it is comparable to the $\gamma$ relaxation observed in ionene glasses by mechanical ${ }^{32-34)}$ and dielectric ${ }^{2)}$ spectroscopy. The $\gamma$-relaxation is assigned to fluctuations of the methylene groups which are transmitted to some extent to the polar ammonium group, which makes this relaxation dielectrically active. The strong dependence on the length of the spacer fits well into this interpretation.

The influence of the amount of salt added to the polymers on the dielectric function was studied for the polymer P3. This polymer is known to form a poorly ordered lamellar superstructure due to microphase separation of the zwitterionic and the alkyl fragments ${ }^{20,21)}$, which is modified upon mixing with inorganic salts ${ }^{19,24,25)}$. Equi- 
Tab. 1. Fit parameters of the relaxation process for different salt concentrations according to Eq. (2)

\begin{tabular}{cccccc}
\hline $\begin{array}{l}\text { Concen- } \\
\text { tration } \\
\text { in mol-\% }\end{array}$ & $\begin{array}{l}\text { Temp. } \\
\text { in } \mathrm{K}\end{array}$ & $\Delta \varepsilon_{\mathrm{HN}}$ & $\alpha_{\mathrm{HN}}$ & $\gamma_{\mathrm{HN}}$ & $\tau_{\mathrm{HN}}$ \\
\hline \multirow{2}{*}{0} & & & & & \\
& 214 & 1,904 & 0,420 & 0,524 & $9,2 \cdot 10^{-5}$ \\
& 224 & 1,692 & 0,475 & 0,524 & $1,8 \cdot 10^{-5}$ \\
& 233 & 1,762 & 0,465 & 0,524 & $6,7 \cdot 10^{-6}$ \\
& 243 & 1,684 & 0,506 & 0,524 & $2,6 \cdot 10^{-6}$ \\
& 253 & 1,684 & 0,530 & 0,524 & $1,1 \cdot 10^{-6}$ \\
100 & 262 & 1,783 & 0,502 & 0,524 & $5,7 \cdot 10^{-7}$ \\
& 202 & 0,599 & 0,475 & 0,444 & $8,4 \cdot 10^{-5}$ \\
& 212 & 0,617 & 0,488 & 0,444 & $3,0 \cdot 10^{-5}$ \\
& 221 & 0,644 & 0,489 & 0,444 & $1,2 \cdot 10^{-5}$ \\
& 231 & 0,610 & 0,539 & 0,444 & $4,8 \cdot 10^{-6}$ \\
& 241 & 0,562 & 0,604 & 0,444 & $1,8 \cdot 10^{-6}$ \\
& 250 & 0,529 & 0,656 & 0,444 & $5,5 \cdot 10^{-7}$ \\
& 260 & 0,549 & 0,643 & 0,444 & $5,6 \cdot 10^{-7}$ \\
& 193 & 1,245 & 0,454 & 0,529 & $7,0 \cdot 10^{-5}$ \\
& 203 & 1,299 & 0,458 & 0,529 & $2,5 \cdot 10^{-5}$ \\
& 212 & 1,266 & 0,491 & 0,529 & $8,8 \cdot 10^{-6}$ \\
& 222 & 1,239 & 0,521 & 0,529 & $1,5 \cdot 10^{-6}$ \\
& 232 & 1,196 & 0,555 & 0,529 & $7,7 \cdot 10^{-6}$ \\
& 241 & 1,215 & 0,558 & 0,529 & $4,3 \cdot 10^{-7}$ \\
& 251 & 1,270 & 0,542 & 0,529 & $10^{-7}$
\end{tabular}

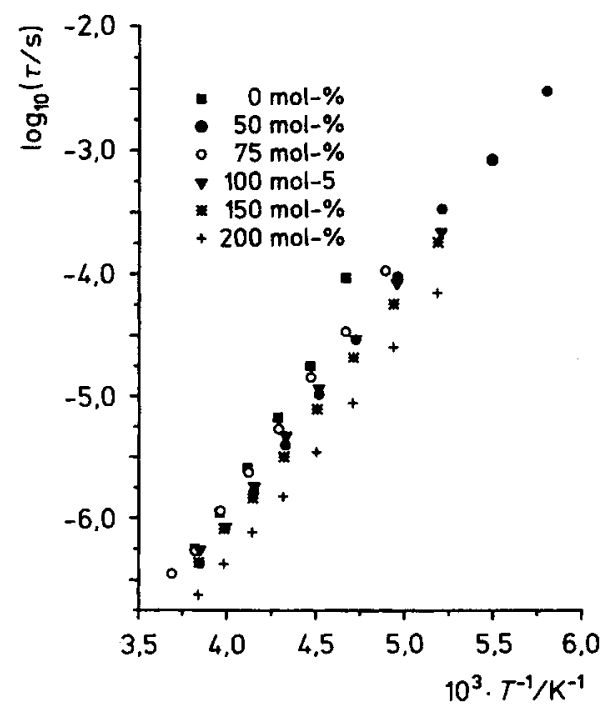

Fig. 4. Activation plot of the $\gamma$ relaxation for different

concentrations of $\mathrm{NaI}$ admixed to polymer $\mathbf{P 3}$ 
molar mixtures of $\mathrm{P3}$ with $\mathrm{NaI}$ are homogeneous according to X-ray scattering studies, but mixtures containing $150 \mathrm{~mol}-\%$ of $\mathrm{NaI}$ exhibit the presence of phase-separated excess $\mathrm{NaI}^{24)}$. While the pure polymer $\mathrm{P3}$ is stable upto about $483 \mathrm{~K}$, mixtures with $\mathrm{NaI}$ start to decompose already at much lower temperatures of above $453 \mathrm{~K}$ due to the strong nucleophilicity of the iodide anion ${ }^{24)}$. Fig. 5 shows the complex dielectric function $\varepsilon^{*}(\omega)$ as a function of frequency for different concentrations of $\mathrm{NaI}$ added. The real $\varepsilon^{\prime}$ and imaginary $\varepsilon^{\prime \prime}$ parts of the dielectric function exhibit a gradual increase with increasing salt concentration and the increasing contribution of the conductivity. The increase of $\varepsilon^{\prime}$ is caused by electrode polarization of the ionic charge carriers.

To analyse the extent of the electrode polarization effect, an Argand representation of the complex impedance $Z$ is used (Fig. 6), $Z=Z^{\prime}+\mathrm{i} Z^{\prime \prime}$, with

$$
\begin{aligned}
& Z^{\prime}=\left(1 / \omega C_{0}\right)\left(\varepsilon^{\prime \prime} /\left(\varepsilon^{\prime 2}+\varepsilon^{\prime 2}\right)\right) \\
& Z^{\prime \prime}=\left(1 / \omega C_{0}\right)\left(\varepsilon^{\prime} /\left(\varepsilon^{\prime 2}+\varepsilon^{\prime 2}\right)\right)
\end{aligned}
$$

where $C_{0}$ is the capacitance of the empty condenser. Fig. 6 shows decreasing real $Z^{\prime}$ and imaginary $Z^{\prime \prime}$ parts of the complex impedance with increasing salt concentration and increasing deviations on the low frequency side with increasing $\mathrm{NaI}$ concentration. This effect becomes more pronounced with increasing both temperature and salt concentration, and hence increasing mobility and effective number density of the charge carriers ${ }^{35}$.
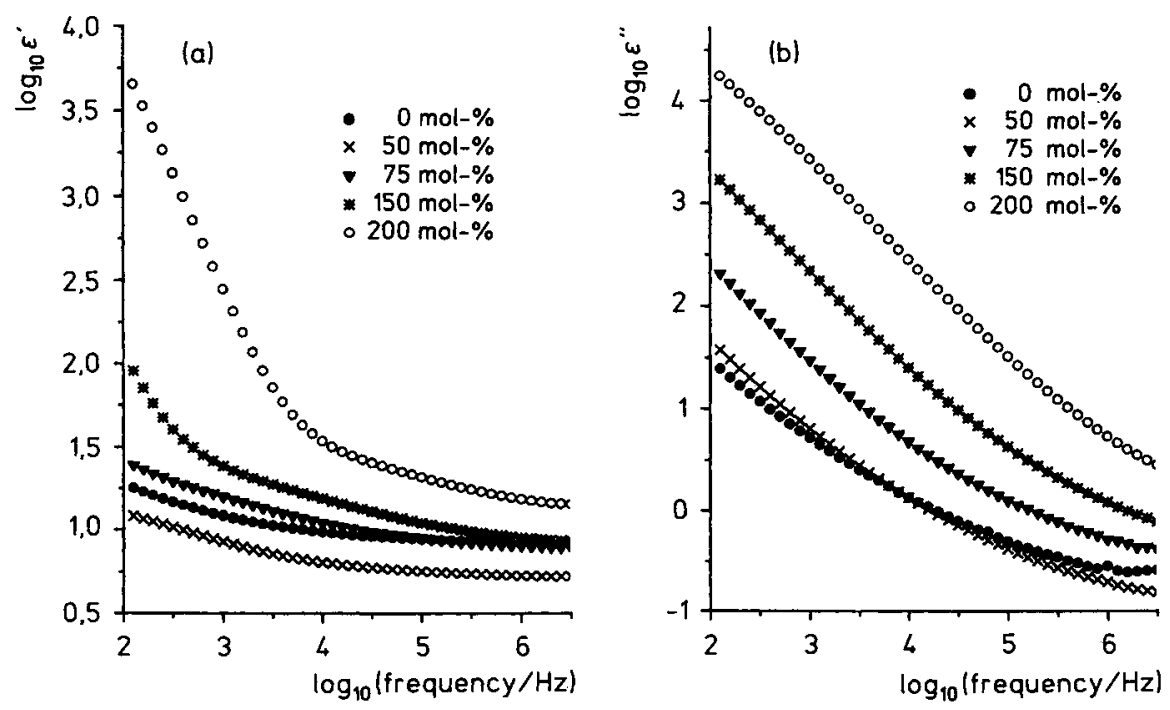

Fig. 5. (a) Real $\varepsilon^{\prime}$ and (b) imaginary $\varepsilon^{\prime \prime}$ parts of the complex dielectric function $\varepsilon^{*}(\omega)$ versus frequency for $\mathbf{P 3}$ at different concentrations of $\mathrm{NaI}$ added $(T=396 \mathrm{~K})$ 


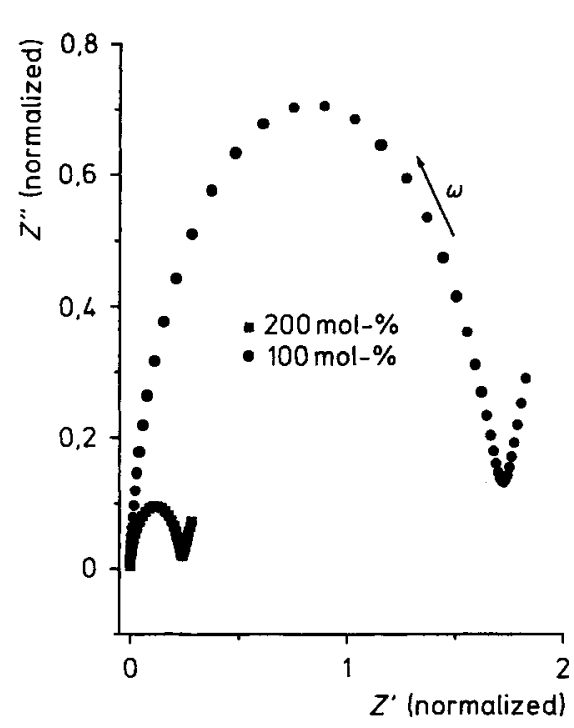

Fig. 6.

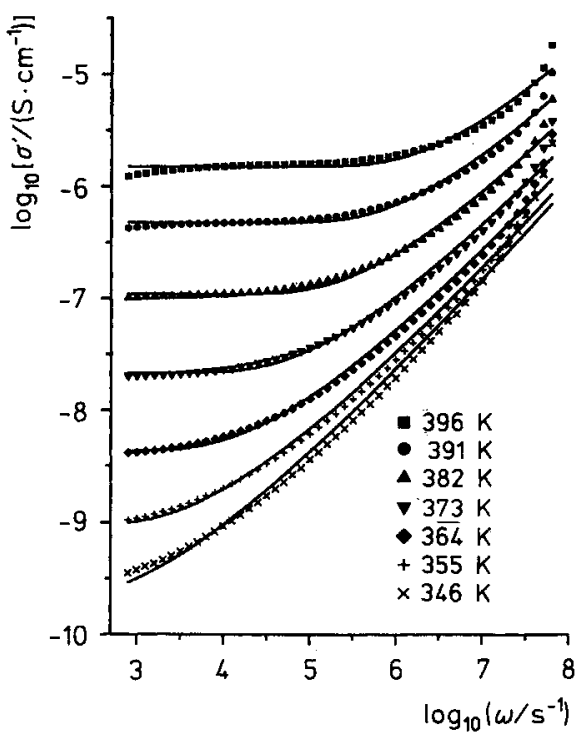

Fig. 7.

Fig. 6. Argand plots of the complex normalized impedance $Z$ for polymer $\mathbf{P 3}$ with different concentrations of the $\mathrm{NaI}(T=396 \mathrm{~K})$. Normalization factor $=10 \mathrm{k} \Omega$ for $Z^{\prime}$ and $Z^{\prime \prime}$

Fig. 7. Real $\sigma^{\prime}$ part of the complex conductivity versus frequency for $\mathbf{P} 3$ with $200 \mathrm{~mol}-\%$ of $\mathrm{NaI}$ added at different temperatures. The solid lines are fitted using Eq. (4)

The frequency and temperature dependence of the conductivity (Fig. 7) of the saltpolyzwitterion mixtures is characterized quantitatively by the following features: (i) The conductivity increases with increasing frequency and temperature; (ii) for high temperatures, the conductivity is almost constant at low frequencies but increases rapidly at a certain critical frequency $\omega_{c}$; (iii) this critical frequency shifts to lower values with decreasing temperature; (iv) above this critical frequency, the conductivity is proportional to $\omega^{\mathrm{s}}$ with $0,7 \leq s \leq 1$; (v) the exponent $s$ increases as the temperature decreases, approximating 1 ; (vi) the conductivity increases with increasing $\mathrm{NaI}$ concentration (Fig. 8a), showing a frequency dependence similar to the observed temperature dependence; (vii) for the imaginary part of the conductivity (Fig. 8 b), a power law behaviour is found, with deviations on the low frequency side which are caused by electrode polarization effects.

Among the vast number of theoretical treatments ${ }^{6-18)}$ of charge transport in disordered solids, the random free-energy barrier model for ac conduction by Dyre $^{11-14)}$ is probably worked out best. This model assumes that conduction takes place by hopping, where the hopping charge carriers are subject to spatially randomly varying energy barriers. The model is solved in the continuous time random walk approximation (CTRW). In a disordered material where charge transport takes place between localized states, the energy-level difference as well as the spatial distance can 

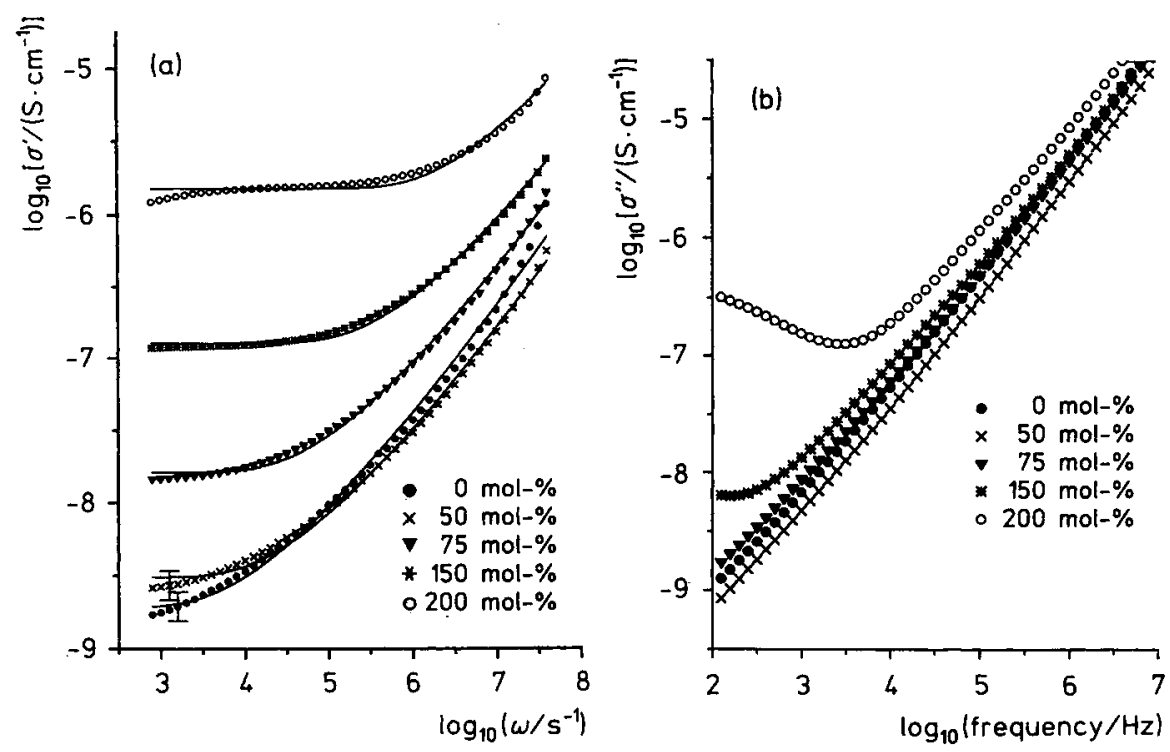

Fig. 8. (a) Real $\sigma^{\prime}$ and (b) imaginary $\sigma^{\prime \prime}$ parts of the complex conductivity versus frequency for the sample P3 at different concentrations of the NaI salt $(T=396 \mathrm{~K})$. The solid lines are fitted using Eq. (4)

vary from site to site. In the CTRW theory, the transport is assumed to take place on a regular lattice. The disorder is taken into account by a distribution of waiting times between successive jumps. The real part of the conductivity in the random free-energy barrier model solved within CTRW approximation is given by ${ }^{11)}$ :

$$
\left.\sigma^{\prime}(\omega)=\sigma_{0}\left[(\omega \tau) \arctan (\omega \tau) /\left(\ln \left[1+(\omega \tau)^{2}\right]^{1 / 2}\right\}^{2}+[\arctan (\omega \tau)]^{2}\right)\right]
$$

where the dc conductivity $\sigma_{0}$ and the time $\tau$ (which will be interpreted later) are fit parameters. The fit describes well the observed frequency, temperature and salt concentration dependences (Fig. 7, 8a).

Eq. (4) has the character of a master equation, because its frequency dependence normalized with respect to $\tau$ occurs only within the rectangular brackets. To verify whether the data follow this scaling behaviour, the measured conductivity is normalised at each temperature resp. salt concentration by the fit parameter $\sigma_{0}$ and plotted against the frequency normalised with respect to the fit parameter $\tau$. For both the temperature (Fig. 9a) and salt concentration (Fig. 9b) dependence, a scaling according to Eq. (4) is observed within experimental accuracy.

A pronounced Arrhenius-type temperature dependence is observed for the fit parameters $\sigma_{0}$ and $\tau$ (Tab. 2, Fig. $10 \mathrm{a}, \mathrm{b}$ ). The product of $\sigma_{0} \tau$ however is temperature independent within the limits of experimental accuracy (Tab. 3, Fig. 11). A fixed proportionality exists between the inverse relaxation time $1 / \tau$ and the critical frequency 

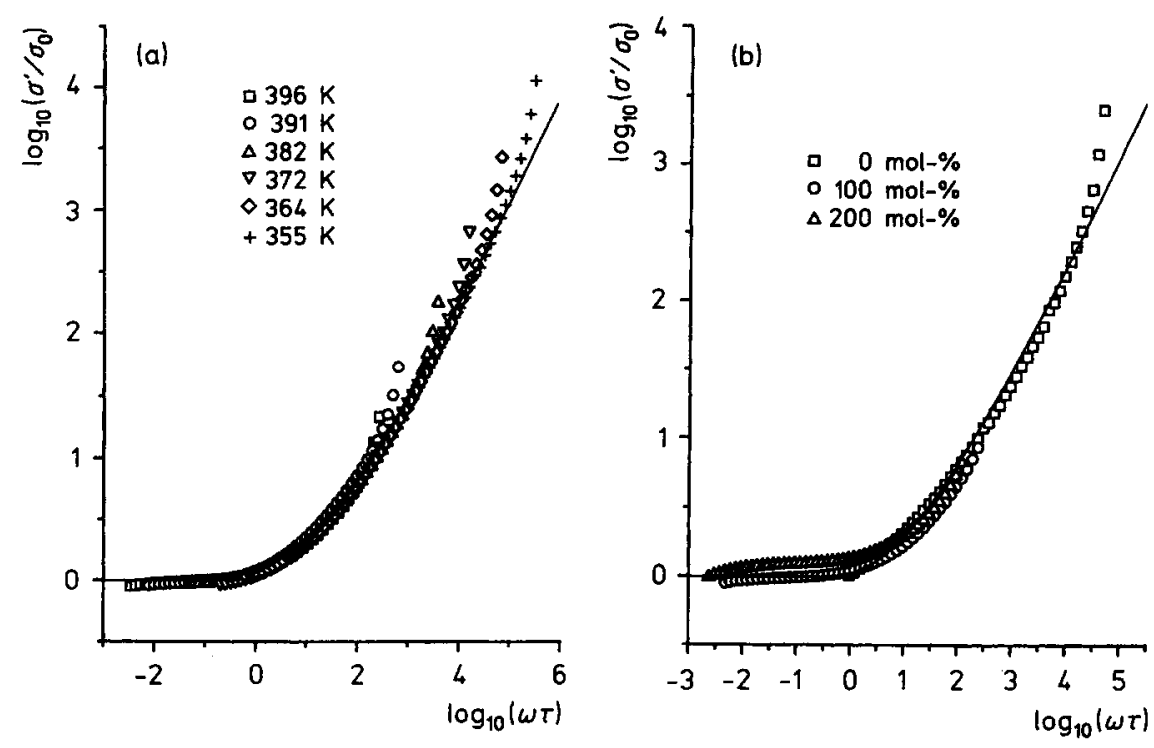

Fig. 9. Master plot of the normalized conductivity versus the normalized frequency for polymer $\mathbf{P 3}$ at (a) various temperatures and (b) various concentrations of added $\mathrm{NaI}$. The line results from Eq. (4)

Tab. 2. Activation energies $E_{\mathrm{A}}$ of the dc conductivity $\sigma_{0}$ and $\tau$ for different salt concentrations

\begin{tabular}{ccccc}
\hline Concentration in mol-\% & $E_{\mathrm{A}} / \mathrm{eV}$ & $\left(\sigma_{0}\right)$ & $E_{\mathrm{A}} / \mathrm{eV}$ & $(1 / \tau)$ \\
\hline 0 & 2,38 & & 2,41 & \\
100 & 2,66 & & 2,68 & \\
200 & 2,09 & & 1,91
\end{tabular}

$\omega_{s}$ which marks the onset of dispersion of the conductivity versus frequency (Tab. 4). Hence, from the constancy of $\sigma_{0} \tau$ the proportionality between $\sigma_{0}$ and $\omega_{\mathrm{s}}$ follows. This is the well known BNN relation according to Barton, Nakajima and Namikawa ${ }^{36-38)}$. It reflects the fact that $\mathrm{dc}$ and ac conduction both occur by the same mechanism.

With decreasing temperature the frequency expontent $s$ increases from almost 0,74 at $T=396 \mathrm{~K}$ to about 1 at $T=346 \mathrm{~K}$ (Fig. 12). The parameter $s$ decreases with increasing salt concentration. For $\omega \tau \gg 1$ the exponent $s$ is given by the expression ${ }^{11)}$

$$
s=1-2 / \ln (\omega \tau)
$$

For $10^{2}<\omega \tau<10^{16}$ the model predicts exponents between 0,62 and $0,95^{11)}$. Since $s \rightarrow 1$ as $\omega \tau \rightarrow \infty$, the model thus predicts $s \rightarrow 1$ as $T \rightarrow 0$, which is in agreement with the data. 
Tab. 3. Fit parameters $\sigma_{0}$ and $\tau$ as deduced from fits according to the Eq. (4), at a salt concentration of 0,100 and $200 \mathrm{~mol}-\% \mathrm{NaI}$

\begin{tabular}{|c|c|c|c|c|c|c|c|c|c|}
\hline \multirow{2}{*}{$\begin{array}{l}\text { Temp. } \\
\text { in } K\end{array}$} & $\sigma_{0} /(\mathrm{S} / \mathrm{cm})$ & $\tau / \mathrm{s}$ & $\sigma_{0} \cdot \tau$ & $\sigma_{0} /(\mathrm{S} / \mathrm{cm})$ & $\tau / \mathrm{s}$ & $\sigma_{0} \cdot \tau$ & $\sigma_{0} /(\mathrm{S} / \mathrm{cm})$ & $\tau / \mathrm{s}$ & $\sigma_{0} \cdot \tau$ \\
\hline & \multicolumn{3}{|c|}{$0 \mathrm{~mol}-\%$} & \multicolumn{3}{|c|}{$100 \mathrm{~mol}-\%$} & \multicolumn{3}{|c|}{$200 \mathrm{~mol}-\%$} \\
\hline 396 & $1,8 \cdot 10^{-9}$ & 0,00075 & $1,36 \cdot 10^{-12}$ & $1,8 \cdot 10^{-7}$ & $3,8 \cdot 10^{-6}$ & $7,03 \cdot 10^{-13}$ & $1,5 \cdot 10^{-6}$ & $2,0 \cdot 10^{-6}$ & $2,98 \cdot 10^{-12}$ \\
\hline 391 & $7,9 \cdot 10^{-10}$ & 0,00157 & $1,24 \cdot 10^{-12}$ & $5,6 \cdot 10^{-8}$ & 0,00001 & $5,55 \cdot 10^{-13}$ & $4,8 \cdot 10^{-7}$ & $4,5 \cdot 10^{-6}$ & $2,16 \cdot 10^{-12}$ \\
\hline 382 & $1,5 \cdot 10^{-10}$ & 0,00755 & $1,16 \cdot 10^{-12}$ & $1,3 \cdot 10^{-8}$ & 0,00004 & $5,30 \cdot 10^{-13}$ & $1,10 \cdot 10^{-7}$ & 0,00001 & $1,10 \cdot 10^{-12}$ \\
\hline 373 & $1,0 \cdot 10^{-11}$ & 0,13636 & $1,40 \cdot 10^{-12}$ & $2,5 \cdot 10^{-9}$ & 0,00018 & $4,49 \cdot 10^{-13}$ & $2,2 \cdot 10^{-8}$ & 0,00006 & $1,30 \cdot 10^{-12}$ \\
\hline 364 & $2,9 \cdot 10^{-12}$ & 0,42599 & $1,22 \cdot 10^{-12}$ & $3,5 \cdot 10^{-10}$ & 0,00129 & $4,48 \cdot 10^{-13}$ & $4,3 \cdot 10^{-9}$ & 0,00027 & $1,17 \cdot 10^{-12}$ \\
\hline 355 & $2,7 \cdot 10^{-13}$ & 5 & $1,47 \cdot 10^{-12}$ & $2,5 \cdot 10^{-11}$ & 0,02331 & $5,93 \cdot 10^{-13}$ & $9,4 \cdot 10^{-10}$ & 0.00119 & $1,12 \cdot 10^{-12}$ \\
\hline 346 & $1,4 \cdot 10^{-13}$ & 10 & $1,44 \cdot 10^{-12}$ & $1,7 \cdot 10^{-12}$ & 0,41942 & $7,39 \cdot 10^{-13}$ & $2,0 \cdot 10^{-10}$ & 0,00606 & $1,20 \cdot 10^{-12}$ \\
\hline
\end{tabular}

Tab. 4. Comparison between fit parameter $\tau$ and critical frequency $\omega_{\mathrm{s}}$ deduced from experimental data by calculating numerically the second derivative of the conductivity vs. frequency (salt conc.: 100 and $200 \mathrm{~mol}-\% \mathrm{NaI}$ )

\begin{tabular}{|c|c|c|c|c|c|c|}
\hline \multirow{2}{*}{$\begin{array}{l}\text { Temp. } \\
\text { in } K\end{array}$} & $\omega_{\mathrm{s}} \cdot \tau$ & $\log _{10}\left(\tau^{-1} / \mathrm{s}^{-1}\right)$ & $\log _{10} \omega_{\mathrm{s}}$ & $\omega_{s} \cdot \tau$ & $\log _{10}\left(\tau^{-1 / s^{-1}}\right)$ & $\log _{10} \omega_{s}$ \\
\hline & \multicolumn{3}{|c|}{$200 \mathrm{~mol}-\%$} & \multicolumn{3}{|c|}{$100 \mathrm{~mol}-\%$} \\
\hline 396 & 0,20 & 5,70 & 5,00 & 0,12 & 5,41 & 4,50 \\
\hline 391 & 0,22 & 5,35 & 4,70 & 0,16 & 5,00 & 4,20 \\
\hline 382 & 0,08 & 5,00 & 3,90 & 0,10 & 4,40 & 3,40 \\
\hline 373 & 0,12 & 4,22 & 3,30 & 0,28 & 3,74 & 3,20 \\
\hline 364 & 0,17 & 3,57 & 2,80 & 1,28 & 2,89 & 3,00 \\
\hline
\end{tabular}



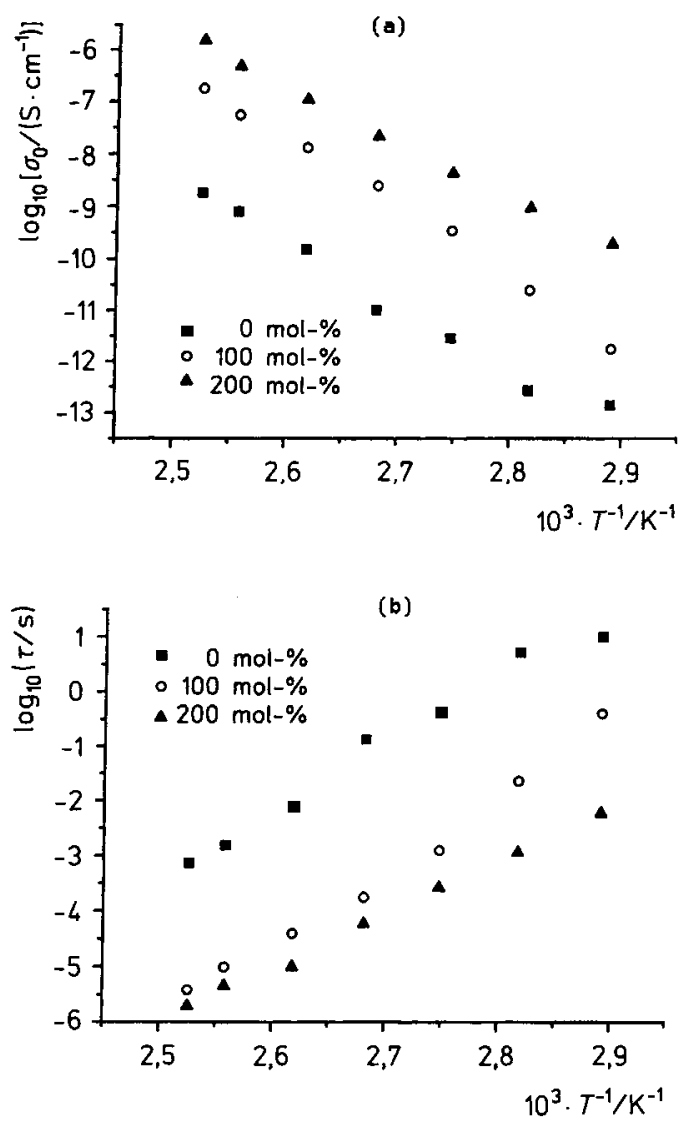

Fig. 10. Temperature dependence of the fit parameters $\sigma_{0}$ (a) and $\tau$ (b) for different concentrations of Nal added to polymer P3

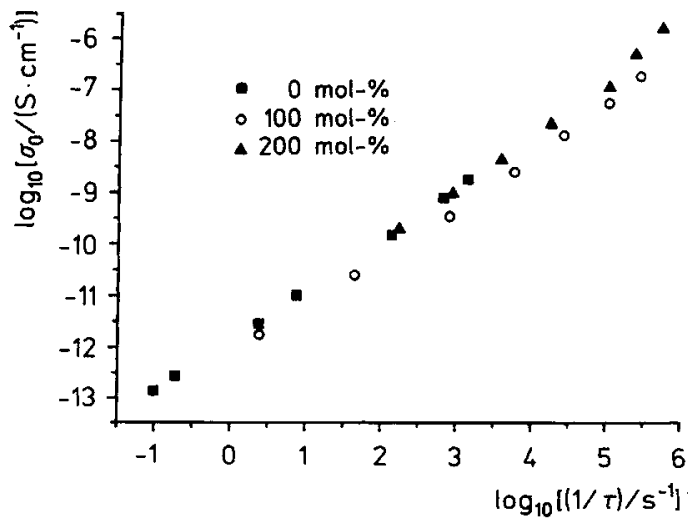

Fig. 11. A log-log plot of dc conductivity $\sigma_{0}$ against the inverse relaxation time $1 / \tau$ for different $\mathrm{NaI}$ concentrations in $\mathbf{P 3}$ 
Fig. 12. Temperature and concentration dependence of the expontent $s$ for mixtures of $\mathbf{P 3}$ and $\mathrm{NaI}$

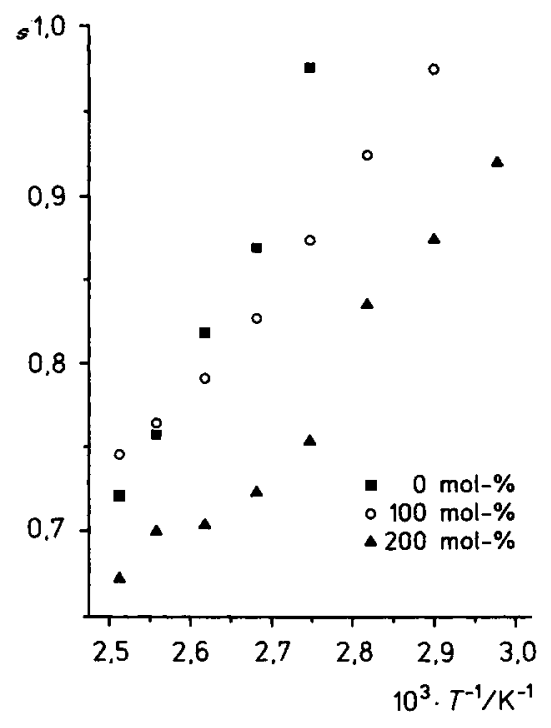

\section{Conclusions}

The dielectric properties of mixtures of the inorganic salt $\mathrm{NaI}$ and a polyzwitterion were measured in the frequency range from $10^{2} \mathrm{~Hz}$ to $10^{7} \mathrm{~Hz}$ and at temperatures between $110 \mathrm{~K}$ and $400 \mathrm{~K}$ for different salt concentrations $(0 \mathrm{~mol}-\%, 100 \mathrm{~mol}-\%, 200$ mol-\%). Besides a dominating conductivity contribution one relaxation process is observed. It is assigned to fluctuations of the quaternary ammonium in the side group ( $\gamma$-relaxation) and depends in its dynamics sensitively on the length of the aliphatic spacer between the acrylate main chain and the betaine group. The ion concentration has a negligible influence on this relaxation process. The conductivity contribution can be quantitatively understood in its frequency, temperature and ion concentration dependence by hopping of charge carriers in a random free-energy barrier model. For the critical frequency (marking the onset of dispersion of the conductivity versus frequency) and the dc conductivity, a proportionality is observed according to the Barton-Nakajima-Namikawa (BNN) relationship.

A scholarship to S. A. R. from the Volkswagen Foundation is gratefully acknowledged. Support by the German Science Foundation (SFB 262 and SFB 294) and by the Fonds der Chemischen Industrie is highly appreciated. F. K. would like to thank the Max-Planck-Institute for generous support in building up new dielectric facilities at the University of Leipzig. 
1) A. Zetsche, F. Kremer, W. Jung, H. Schulze, Polymer 31, 1883 (1990)

2) F. Kremer, L. Dominguez, W. H. Meyer, G. Wegner, Polymer 30, 2023 (1989)

3) R. R. Rietz, W. H. Meyer, Polymers For Advanced Technologies 4, 164 (1993)

4) F. Kremer, J. Klee, Polymer 33, 3564 (1992)

5) S. L. Hutton, I. Fehst, R. Böhmer, W. Brune, B. Mertz, P. Lunkenheimer, A. Loidl, Phys. Rev. Lett. 66, 1990 (1991)

6) S. R. Elliott, Adv. Phys. 36, 135 (1987)

7) A. K. Jonscher, "Dielectric Relaxation in Solids", Chelsea Dielectric, London 1983

8) D. P. Aimond, C. C. Hunter, A. R. West, J. Mater. Sci. 19, 3236 (1984)

9) L. A. Dissado, R. M. Hill, Phys. Rev. B 37, 3434 (1988)

10) H. Böttger, U. V. Bryskin, "Hopping Conduction in Solids", Akademie Verlag, Berlin 1986

11) J. C. Dyre, Phys. Lett. 108 A, 457 (1985)

12) J. C. Dyre, J. Non-Cryst. Solids 88, 271 (1986)

13) J. C. Dyre, J. Appl. Phys. 64, 2456 (1988)

14) J. C. Dyre, Phys. Rev. B 48, 12511 (1993)

15) K. Funke, R. Hoppe, Solid State Ionics 40/41, 200 (1990)

16) J. R. Macdonald, Solid State Ionics 15, 159 (1985)

17) J. R. Macdonald, J. Appl. Phys. 65, 4845 (1989)

18) J. R. Macdonald, J. Appl. Phys. 62, RI (1987)

19) P. Köberle, A. Laschewsky, T. D. Lomax, Makromol. Chem., Rapid Commun. 12, 427 (1991)

20) P. Köberle, A. Laschewsky, V. Tsukruk, Makromol. Chem. 193, 1815 (1992)

21) V. Tsukruk, N. Mischenko, P. Köberle, A. Laschewsky, Makromol. Chem. 193, 1829 (1992)

22) E. A. Bekturov, S. E. Kudaibergenov, S. R. Rafikov, J. Macromol. Sci., Rev. Macromol. Chem. Phys. C30, 233 (1990)

23) M. Galin, E. Marchal, A. Mathis, B. Meurer, Y. M. Monroy Soto, J. C. Galin, Polymer 28, 1937 (1987)

24) P. Köberle, A. Laschewsky, Macromolecules 27, 2165 (1994)

25) P. Köberle, A. Laschewsky, Makromol. Symp., in press

26) G. C. Bazuin, Y. L. Zheng, R. Muller, J. C. Galin, Polymer 30, 654 (1989)

27) M. Ehrmann, R. Muller, J. C. Galin, C. G. Bazuin, Macromolecules 27, 4910 (1993)

28) J. Cardoso, A. Huanosta, O. Manero, Macromolecules 24, 2890 (1991)

29) D. Graiver, M. Litt, E. Baer, J. Polym. Sci., Polym. Chem. Ed. 17, 3607 (1979)

30) I. Diaconu, P. Coman, T. Buruiana, E. C. Buruiana, Angew. Makromol. Chem. 213, 73 (1993)

31) S. Havriliak, S. Negami, Polymer 8, 161 (1967)

32) T. Tsutsui, T. Sato, T. Tanaka, J. Polym. Sci., Polym. Phys. Ed. 13, 2091 (1975)

33) T. Tsutsui, R. Tanaka, T. Tanaka, J. Polym. Sci., Polym. Phys. Ed. 14, 2259 (1976)

34) T. Tsutsui, R. Tanaka, T. Tanaka, J. Polym. Sci., Polym. Phys. Ed. 14, 2273 (1976)

35) J. R. Macdonald, "Impedance Spectroscopy", John Wiley, New York 1987

36) J. L. Barton, Verres Refra. 20, 328 (1966)

37) T. Nakajima, 1971 Annual Report, Conference on Electric Insulation and Dielectric Phenomena (National Academy of Sciences, Washington DC 1972), p. 168

38) H. Namikawa, J. Non-Cryst. Solids 18, 173 (1975) 